\title{
Probing the structure and function of an archaeal C/D-box methylation guide sRNA
}

\author{
ARINA D. OMER, ${ }^{1}$ MARIA ZAGO, ${ }^{1}$ ALEX CHANG, ${ }^{1}$ and PATRICK P. DENNIS ${ }^{2}$ \\ ${ }^{1}$ Department of Biochemistry and Molecular Biology, University of British Columbia, Vancouver, Canada V6T1Z3 \\ ${ }^{2}$ Division of Molecular and Cellular Biosciences, National Science Foundation, Arlington, Virginia 22230, USA
}

\begin{abstract}
The genome of the hyperthermophilic archaeon Sulfolobus solfataricus contains dozens of small C/D-box sRNAs that use a complementary guide sequence to target $2^{\prime}$ - $O$-ribose methylation to specific locations within ribosomal and transfer RNAs. The sRNAs are $\sim 50-60$ nucleotides in length and contain two RNA structural kink-turn (K-turn) motifs that are required for assembly with ribosomal protein L7Ae, Nop5, and fibrillarin to form an active ribonucleoprotein (RNP) particle. The complex catalyzes guide-directed methylation to target RNAs. Earlier work in our laboratory has characterized the assembly pathway and methylation reaction using the model sR1 sRNA from Sulfolobus acidocaldarius. This sRNA contains only one antisense region situated adjacent to the D-box, and methylation is directed to position U52 in $16 S$ rRNA. Here we have investigated through RNA mutagenesis, the relationship between the sR1 structure and methylation-guide function. We show that although full activity of the guide requires intact $C / D$ and $C^{\prime} / D^{\prime}$ K-turn motifs, each structure plays a distinct role in the methylation reaction. The C/D motif is directly implicated in the methylation function, whereas the $C^{\prime} / D^{\prime}$ element appears to play an indirect structural role by facilitating the correct folding of the RNA. Our results suggest that L7Ae facilitates the folding of the K-turn motifs (chaperone function) and, in addition, is required for methylation activity in the presence of Nop5 and Fib.
\end{abstract}

Keywords: C/D box RNA; 2'-O-ribose methylation; Archaea; K-turn

\section{INTRODUCTION}

The nucleolus is a specialized structure located within the nucleus of the eukaryotic cell that serves as a factory for the production of ribosomes (Hernandez-Verdun 2005). This factory contains dozens of small nucleolar ribonucleoprotein (snoRNP) machines, many of which have been implicated in the endonucleolytic processing, folding, and modification of the precursor ribosomal RNA (rRNA) prior to its assembly into mature ribosomal subunits (Gerbi 1995; Lafontaine and Tollervey 1995; Maxwell and Fournier 1995). The snoRNAs in these machines fall into two distinct classes-the box C/D RNAs and the box H/ACA RNAs - and use complementary guide sequences to direct either 2'-O-ribose methylation or pseudouridylation modification to specific locations within rRNA (Bachellerie et al. 1995; Tollervey and Kiss 1997; Kiss 2001). Our previous work used biochemical and informatics techniques to

Reprint requests to: Arina D. Omer, Department of Biochemistry and Molecular Biology, University of British Columbia, Vancouver, Canada V6T1Z3; e-mail: arina@interchange.ubc.ca; fax: (604) 822-5227.

Article published online ahead of print. Article and publication date are at http://www.rnajournal.org/cgi/doi/10.1261/rna.31506. identify homologs of the eukaryotic C/D-box snoRNAs in prokaryotic Archaea (Omer et al. 2000).

The archaeal C/D-box sRNAs are $\sim 50-60$ nucleotides (nt) in length and, like their eukaryotic equivalents, contain conserved C (UGAUGA) and D (CUGA) box sequences near the respective $5^{\prime}$ - and $3^{\prime}$-ends of the molecule and second copies of the box sequences (termed $\mathrm{D}^{\prime}$ and $\mathrm{C}^{\prime}$ ) near the center of the molecule (Omer et al. 2000). The C and $\mathrm{D}$ and the $\mathrm{C}^{\prime}$ and $\mathrm{D}^{\prime}$ boxes associate to form a distinct fold in the RNA, termed the kink-turn or K-turn (Watkins et al. 2002). Archaeal sRNAs, when viewed as a group, do not exhibit structural and functional homogeneity; nonetheless, the sR1 sRNA of Sulfolobus acidocaldarius has proven to be a useful model and appears to exhibit most of the features of a canonical archaeal sRNA. Generally, the RNA fold associated with the C/D element exhibits the features of an archetypal K-turn consisting of an asymmetric loop flanked by two short helical structures, designated stems I and II (Watkins et al. 2000). In contrast, the $C^{\prime} / D^{\prime}$ associated K-turn only partially matches this consensus: it consists of a terminal loop closed by one stem, the equivalent to stem II. Nevertheless, both $\mathrm{C} / \mathrm{D}$ and $\mathrm{C}^{\prime} / \mathrm{D}^{\prime} \mathrm{K}$-turns contain the two adjacent sheared G:A base pairs that are the characteristic feature of the K-turn motif and serve as 
binding site for the multifunctional archaeal ribosomal protein L7Ae (Kuhn et al. 2002). It is generally believed that a single copy of the core L7Ae protein binds to each of the two K-turns and nucleates at each site the addition of a heterodimer containing the Nop5 and Fibrillarin (Fib) proteins (Rashid et al. 2003; Tran et al. 2003). The coiledcoil autoassociation domain within Nop5 is believed to stabilize the complex of the RNA with the six protein subunits (Aittaleb et al. 2003). The Fib protein contains an $S$-adenosyl methionine binding domain that is located at the interface with the Nop5 protein; mutational analysis has identified Fibrillarin as the methyltransferase in the modification reaction (Omer et al. 2002).

The guide sequences in the sRNA are located immediately upstream of the start of the $\mathrm{D}^{\prime}$ or D-box sequences and are generally complementary to rRNA sequences over a range of $\sim 9-11 \mathrm{nt}$; methylation is directed to the nucleotide in the rRNA target that forms a Watson-Crick base pair $5 \mathrm{nt}$ upstream of the start of the $\mathrm{D}^{\prime}$ of the D-box guide. This is the "N plus five" rule (Bachellerie and Cavaille 1997; Filipowicz et al. 1999; Weinstein and Steitz 1999). Work in our laboratory first demonstrated that these complexes can be assembled using an RNA transcribed in vitro and purified recombinant proteins. When provided with a suitable complementary target RNA and $S$-adenosyl methionine, these complexes are active in directing site-specific ribose methylation in vitro (Omer et al. 2002).

The eukaryotic C/D-box snoRNPs are slightly more complex. They contain a core K-turn binding protein $(15.5 \mathrm{kDa}$ in humans or Snu13p in yeast) that is a paralog to the eukaryotic ribosomal proteins L7Ae, L30, and S12 (Vidovic et al. 2000; Watkins et al. 2002). The C/Dbox-associated protein is also multifunctional and is a component of the U4 snRNP that functions in the spliceosome to remove intronic sequences from eukaryotic mRNAs (Vidovic et al. 2000; Nottrott et al. 2002). In addition, the two copies of the Nop5 protein in the archaeal complex are replaced in eukaryotic C/D-box RNP complexes by one copy each of two paralogous proteins, Nop56 and Nop58.

Although there is as yet no complete structure of a fully assembled archaeal or eukaryotic C/D-box sRNP complex, there are a number of solved structures for the $15.5-\mathrm{kDa}$ protein from human (Vidovic et al. 2000), the Snu13p from yeast (Vidovic et al. 2000), and the L7Ae protein from Archaea in complex with various RNA K-turn motifs as well as structures for the archaeal Fibrillarin and the FibrillarinNop5 dimer (Hamma and Ferre-D’Amare 2004; Moore et al. 2004; Suryadi et al. 2005). The cocrystal structure of the Archaeoglobus fulgidus L7Ae bound to a C/D-box RNA oligonucleotide revealed the details of the interaction between the protein and a terminal K-turn motif. Within the crystal, L7Ae contacts six nucleotide positions. One of these is U5 (numbering system as in Moore et al. 2004; see Fig. 1D,E) at the base of stem II; this position is part of a U$\mathrm{U}$ mismatch pair that is conserved in most eukaryotic and archaeal C/D-box representatives and has been postulated to play an essential role in the assembly of higher-order complexes (Watkins et al. 2000, 2002; Szewczak et al. 2005). Of the remaining five contacts, two map to the Gs (G6 and G19) present in the shared G:A-A:G base pairs and three to the unpaired nucleotides located in the asymmetric loop, including a conserved $U$ that protrudes from the RNA backbone into a hydrophobic pocket of the protein (Moore et al. 2004). In a second example, the cocrystal structure of Methanococcus jannaschii L7Ae bound to an internal K-turn that is flanked by a single stem has been solved (Suryadi et al. 2005). In Archaea, the K-turn motifs formed by the $C^{\prime} /$ $\mathrm{D}^{\prime}$-box sequences and in H/ACA sRNAs are located at or near the internal central loop. This structure revealed that despite the differences apparent between an internal and
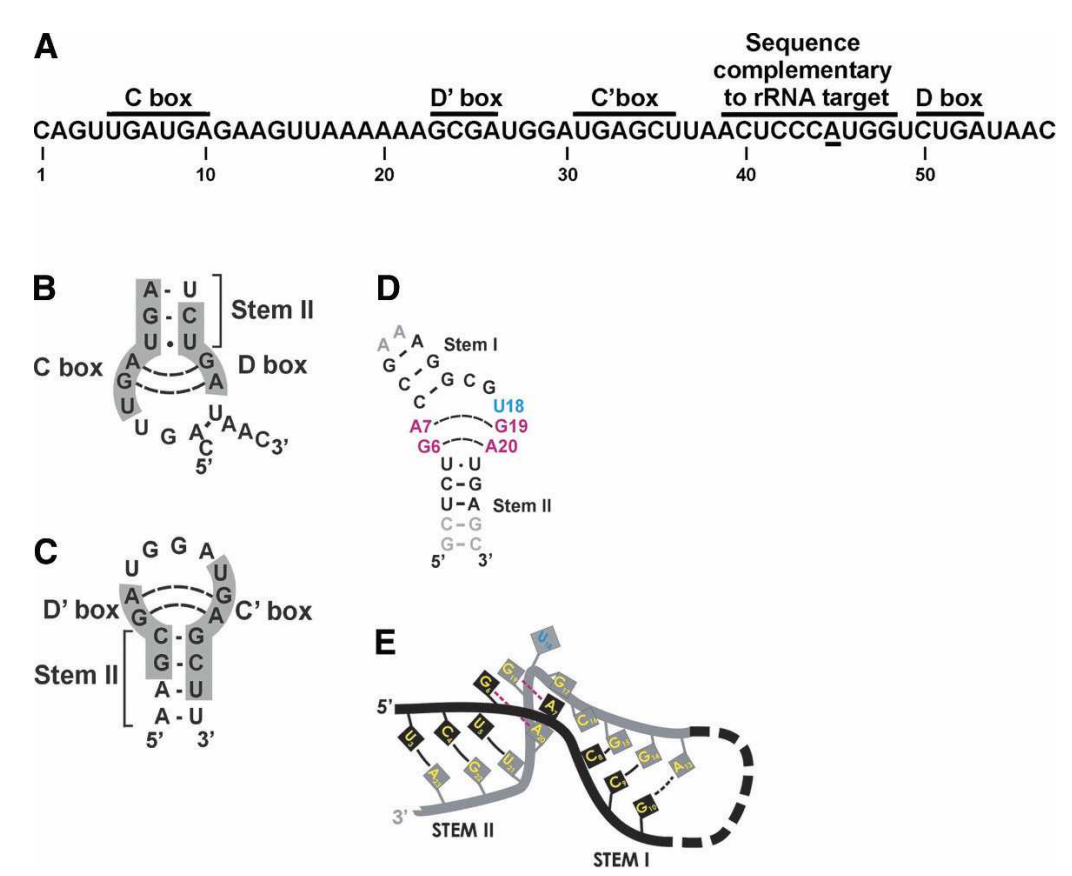

FIGURE 1. Structure of sR1 C/D-box RNA guide from $S$. acidocaldarius. (A) The full-length sequence of sR1 is shown; the C-, $\mathrm{D}^{\prime}-, \mathrm{C}^{\prime}$-, and D-boxes and the guide sequence complementary to the rRNA that directs methylation to position U52 in $16 \mathrm{~S}$ rRNA target are overlined. The A residue in the guide that base pairs with the $\mathrm{U} 52$ residue is underlined. $(B, C)$ Predicted secondary structures of the $S$. acidocaldarius $\mathrm{sR} 1 \mathrm{C} / \mathrm{D}$ and $\mathrm{C}^{\prime} / \mathrm{D}^{\prime} \mathrm{K}$-turn, respectively; the box elements are highlighted and the position of the noncanonical stem II is indicated. $(D, E)$ The secondary and tertiary structures of the RNA K-turn used and solved in the crystallization study by Moore et al. (2004) are depicted (data adapted from Dennis and Omer 2005). 
a terminal canonical K-turn, the same nucleotide residues serve as cues for protein recognition (Hamma and FerreD'Amare 2004).

In this study, we have used site-directed RNA mutagenesis and in vitro assembly and activity assays to examine the structure-function relationships of C/D-box sRNAs. We chose for study the well-characterized model sR1 sRNA from S. acidocaldarius. This sRNA exhibits many of the canonical features of archaeal sRNAs and guides methyl modification to position U52 in $16 \mathrm{~S}$ rRNA, in vivo. In vitro, the sRNA can be efficiently assembled into a complex containing the L7Ae, Nop5, and Fib proteins, and the complex is active in multiple rounds of accurate methylation of a suitable complementary target RNA substrate. Our results show (1) that the sequence and integrity of both the $C / D$ and $C^{\prime} / D^{\prime}$ K-turn motifs in sR1 are required for full activity; and (2) that the sequence and integrity of the two motifs are not functionally equivalent although their positions within the sRNA are interchangeable. In addition, our results suggest that the L7Ae protein facilitates the folding of the K-turn motifs (chaperone function) and as well is required, either directly or indirectly, for methylation activity in the presence of Nop5 and Fib.

\section{RESULTS}

Unlike many euryarchaeal C/D-box RNAs that contain antisense elements upstream of both $\mathrm{D}$ - and $\mathrm{D}^{\prime}$-boxes, the C/D-box RNA homologs encoded by the Crenachaeota branch of Archaea generally contain a single functional guide region that is typically situated upstream of the D element (Omer et al. 2000). The well-characterized $S$. acidocaldarius sR1 sRNA is a representative of the single guide subfamily of C/D-box sRNAs (Figs. 1A-C, 2A, 3A). The RNA contains a 10nt-long guide sequence that is complementary to a region near the $5^{\prime}$-end of the 16S rRNA. The RNP complex assembled with sR1 is active in directing methylation to position U52 in 16S rRNA both in vivo and in vitro (Omer et al. 2002). We have used the in vitro assembly and methylation system to probe by mutagenesis the relationship between structure and function in this sRNA.

\section{Circular permutations of sR1}

In circularly permuted RNAs, the wildtype $5^{\prime}$ - and $3^{\prime}$-termini are connected together, and new extremities are created at alternative positions. Circular permutations have been used as a tool to probe the folding pathway and tertiary structure requirements of various RNAs (Pan et al. 1991; Gott et al. 1993; Harris and Pace 1995). To examine the importance of the position and connectivity of C/D- and $\mathrm{C}^{\prime} / \mathrm{D}^{\prime}$-boxes within the sRNA sequence, we constructed two circularly permuted versions of the $S$. acidocaldarius sR1 RNA (CP1A and CP2) (Figs. 2B,C, 3B,C). In the CP1A sRNA, the $5^{\prime}-$ and $3^{\prime}-$ termini were relocated to a position between the $\mathrm{C}^{\prime}$ and $\mathrm{D}^{\prime}$ box sequences, so that the box C/D K-turn was closed by the internal loop and the $\mathrm{C}^{\prime} / \mathrm{D}^{\prime} \mathrm{K}$-turn loop was opened. In the CP2 sRNA, the $5^{\prime}$ - and $3^{\prime}$-termini were relocated between the $\mathrm{C}$ and $\mathrm{D}^{\prime}$-boxes. As a result, both K-turns in $\mathrm{CP} 2$ are presumably closed by internal loops.

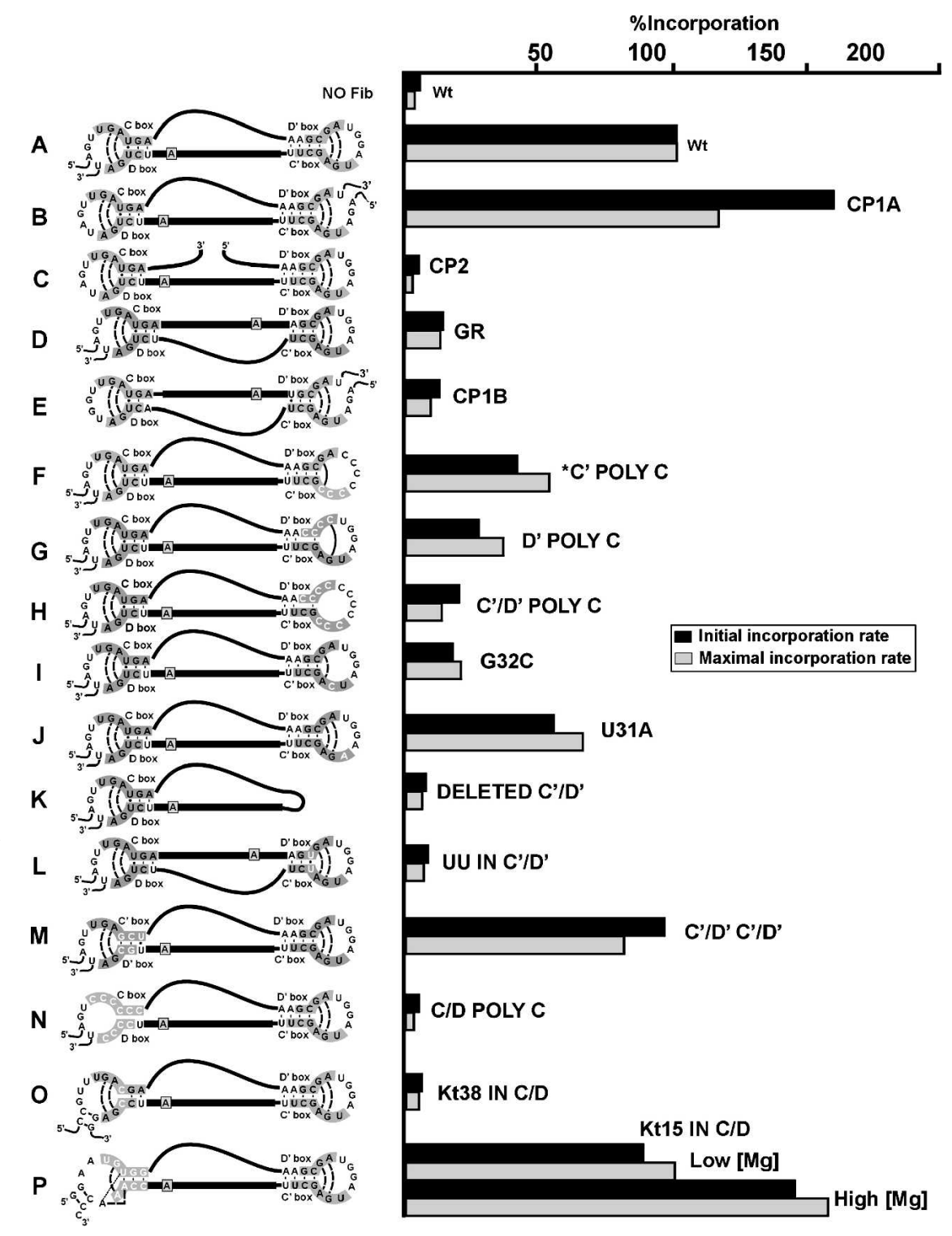

FIGURE 2. (Legend on next page) 
The wild-type and the two permuted sRNAs were analyzed by gel retardation assay for the ability to bind L7Ae, Nop5, and Fib and tested for in vitro methylationguide activity. The gel shift assays indicate that in presence of L7Ae, Nop5, and Fib, both mutants were able to assemble into higher-order RNPs complexes with efficiency comparable to the control sR1 RNA (Fig. 3A-C). However, it was noted that the two mutant sRNAs behaved noticeably differently upon addition of L7Ae; CP1A RNA readily accumulates into Complex I, whereas very little CP2 RNA is able to assemble into Complex I even at high protein concentration (cf. top panels in Fig. 3B,C). Similar variations in the formation of Complex I were sometimes observed with other mutant sRNAs. We suspect that these may be due to the formation of competing structures within the free RNA that are less able to bind the L7Ae protein. In contrast, the mobilities of Complexes II and III formed upon addition of Nop5 and Fib in all instances were comparable to those obtained using wild-type sR1 RNA.

In the presence of $S$-adenosyl-methionine substrate and the complementary target RNA, the circularly permuted RNP complexes exhibited markedly different activities. The CP1A-containing sRNP exhibited slightly elevated methylation activity (measured as initial rate or final level of incorporation) compared to the wild-type sR1-containing control (Figs. 2B, 3B,D). In contrast, repositioning of the $5^{\prime}$ - and 3'-termini between the $\mathrm{C}$ - and $\mathrm{D}^{\prime}$-boxes (CP2) appears to block completely the ability of the complex to direct methyl transfer (the 5\% level of incorporation was equivalent to that observed with sR1-containing complexes that lack the Fib methyltransferase) (Figs. 2C, 3C,D). These results indicate that the guide region can function in association with either a terminal or an internal K-turn formed by the C/D-box sequences; in contrast, the ends of the sRNA cannot be relocated between the C- and $\mathrm{D}^{\prime}$-boxes.
FIGURE 2. Structure and methylation activity of sRNPs containing sR1wild-type and mutant sRNAs. Cartoons on the left section of the figure depict the partial sequence and structure of wild-type and various altered sR1 RNAs. The $5^{\prime}$-terminal $\mathrm{C}$ and the $3^{\prime}$-terminal AAC nucleotides are represented as solid thin line tails. The C-, $\mathrm{D}^{\prime}-, \mathrm{C}^{\prime}-$-, and $\mathrm{D}$-boxes are highlighted in gray, and the two sheared A:G base pairs in each K-turn are indicated by dashed lines across the loop. The D-box guide sequence that is complementary to positions of 49-58 in the $16 \mathrm{~S}$ rRNA is represented as a thick solid line, and the A residue that base pairs with the U52 methylation site in rRNA is boxed. The residues within the box sequences that were altered by mutation are shown in white, and the spacer sequences are represented by solid thin lines. The depicted structures have the following features: $(A)(\mathrm{Wt})$ wild-type sR1; $(B)(\mathrm{CP} 1 \mathrm{~A})$, a circular permutation of sR1 in which the normal $5^{\prime}$ - and $3^{\prime}$-ends of the RNA are joined by ligation between nt A2 and U54, new $3^{\prime}$ - and $5^{\prime}$-ends are introduced between nt U27 and A28, and a $\mathrm{G}(28) \mathrm{A}$ mutation was introduced to provide a base pair to close the terminal loop; $(C)$ (CP2) a circular permutation of sR1 in which the normal $5^{\prime}$ - and $3^{\prime}$-ends of the RNA are joined by ligation between nt A2 and U54 and new $3^{\prime}$ - and $5^{\prime}$-ends are introduced between nt G14 and U15; (D) (GR) guide relocation, a relocation (exchange) of the $\mathrm{D}^{\prime}$ - and D-box guide sequences; $(E)(\mathrm{CP} 1 \mathrm{~B})$ a derivative of $\mathrm{CP} 1 \mathrm{~A}$ in which the $\mathrm{D}^{\prime}$ - and $\mathrm{D}$-box guide sequences were exchanged; $(F)\left({ }^{*} \mathrm{C}^{\prime}\right.$ POLY C) a derivative of sR1 in which the nucleotides between positions 31 and 33 in the $\mathrm{C}^{\prime}$-box were replaced by Cs; $(G)$ ( $\mathrm{D}^{\prime}$ POLY C) a derivative of sR1 in which the nucleotides in the $\mathrm{D}^{\prime}$-box were replaced by Cs; $(H)\left(\mathrm{C}^{\prime} / \mathrm{D}^{\prime}\right.$ POLY C) a derivative of sR1 in which the nucleotides between positions 23 and 33 were replaced by Cs; (I) (G32C) a derivative of sR1 containing a $\mathrm{G}(32) \mathrm{C}$ mutation; $(J)$ (U31A) a derivative of sR1 containing a U(31)A mutation; $(K)$ (DELETED $\left.\mathrm{C}^{\prime} / \mathrm{D}^{\prime}\right)$ a derivative of $\mathrm{sR} 1$ in which the nucleotides between position 24 and 34 are deleted to remove the K-turn formed by the $\mathrm{D}^{\prime}$ - and $\mathrm{C}^{\prime}$-box sequences; $(L)\left(\mathrm{UU}\right.$ in $\left.\mathrm{C}^{\prime} / \mathrm{D}^{\prime}\right)$ a derivative of $\mathrm{sR} 1$ in which the $\mathrm{D}^{\prime}$ - and $\mathrm{D}$-box guide sequences were exchanged and $\mathrm{C} 24 \mathrm{U}$ and $\mathrm{G} 34 \mathrm{U}$ mutations were introduced to produce a $\mathrm{U}-\mathrm{U}$ pair at the top of stem II; $(M)\left(\mathrm{C}^{\prime} / \mathrm{D}^{\prime} \mathrm{C}^{\prime} / \mathrm{D}^{\prime}\right)$ a derivative of sR1 in which the sequences of the $\mathrm{C}$ - and $\mathrm{D}$-boxes were changed so that they were identical to the sequences of the $\mathrm{D}^{\prime}$ - and $\mathrm{C}^{\prime}$-boxes; $(N)(\mathrm{C} / \mathrm{D}$ POLY C) a derivative of sR1 in which all nucleotides in the C- and D-boxes were replaced by Cs; $(O)(\mathrm{Kt} 38 \mathrm{IN} \mathrm{C/D})$ a derivative of $\mathrm{sR} 1$ in which nucleotides between positions 2 and 7 were replaced by CCGUUUGAC and nucleotides between 51 and 55 were replaced by CGAGG, such that the $\mathrm{C} / \mathrm{D} \mathrm{K}$-turn is replaced by K-turn 38 present in the $23 \mathrm{~S}$ rRNA from $\mathrm{H}$. marismortui (Klein et al. 2001); and $(P)(\mathrm{Kt} 15 \mathrm{IN} \mathrm{C} / \mathrm{D})$ a derivative of sR1 in which nucleotides between 1 and 10 were replaced by GGAAUCCGUGG and nucleotides between position 51 and 57 were replaced by CCAAACCC, such that the C/D K-turn was replaced by K-turn 15 present in the $23 \mathrm{~S}$ rRNA from S. solfataricus. The unusual A:U:A base triple in the terminal K-turn 15 is indicated by a dashed line. The right half of the figure indicates the in vitro methylation activity of complexes formed with the various sRNAs in the presence of L7Ae, Nop5, and Fib under standard reaction conditions. The activity of wild-type sR1 was set at $100 \%$, and the activity was assessed either as the initial rate of methyl incorporation (black bar) or as the final plateau in incorporation (gray bar). The activity of the Kt15-containing sRNA $(P)$ was measured under standard low $(1 \mathrm{mM})$ and nonstandard high $(10 \mathrm{mM}) \mathrm{Mg}^{2+}$ concentrations.

\section{Relocation of the guide sequence from the $D$ to the $D^{\prime}$ position}

To test the equivalence of the $C / D$ and $\mathrm{C}^{\prime} / \mathrm{D}^{\prime}$ K-turns, two rearrangements were constructed in which the guide sequence was moved from its normal position adjacent to the D-box to a new position adjacent to the $\mathrm{D}^{\prime}$-box. This was done within the context of both the wild-type and the CP1A sRNAs (Fig. 2D,E). Although both RNAs (GR and CP1B) appear to assemble into higherorder RNP complexes, as measured by the standard gel retardation assay (data not shown), they exhibit $<15 \%$ of the methylation activity of the wild-type sR1 control (Fig. 2D,E). These results indicate that the K-turn formed by the $\mathrm{C}^{\prime} / \mathrm{D}^{\prime}$-box sequences either in the closed (internal) or open (terminal) arrangement is much less efficient in guidedirected methylation than the K-turn formed by the C/D-box sequences in either the closed or open arrangement.

\section{Binding of L7Ae, Nop5, and Fib to the $C^{\prime} / D^{\prime}$ K-turn motif of sR1}

To assess the contribution of the $\mathrm{C}^{\prime} / \mathrm{D}^{\prime}$ motif to RNP complex formation, we generated a basic sRNA unit containing only the $\mathrm{C}^{\prime} / \mathrm{D}^{\prime}$ motif and the adjacent stem II. This minimal RNA structure is able to form a complex with L7Ae, Nop5, and Fib (Fig. 3F). With this 
A

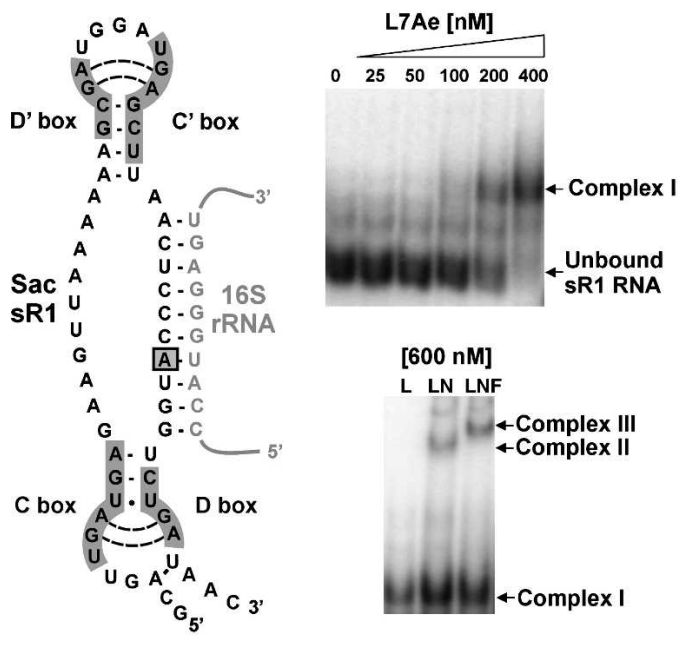

B

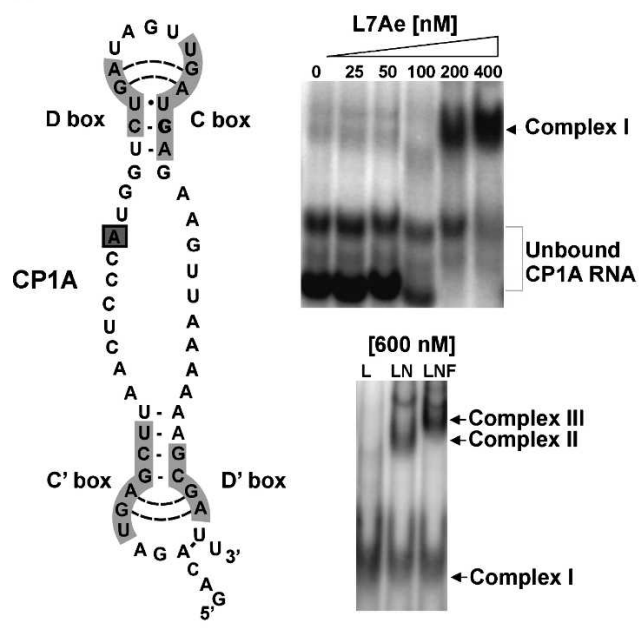

C

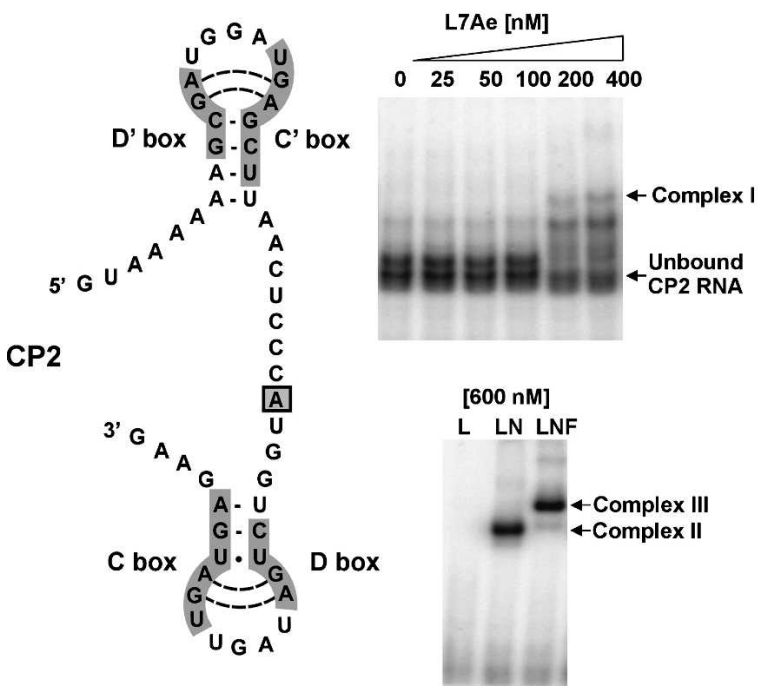

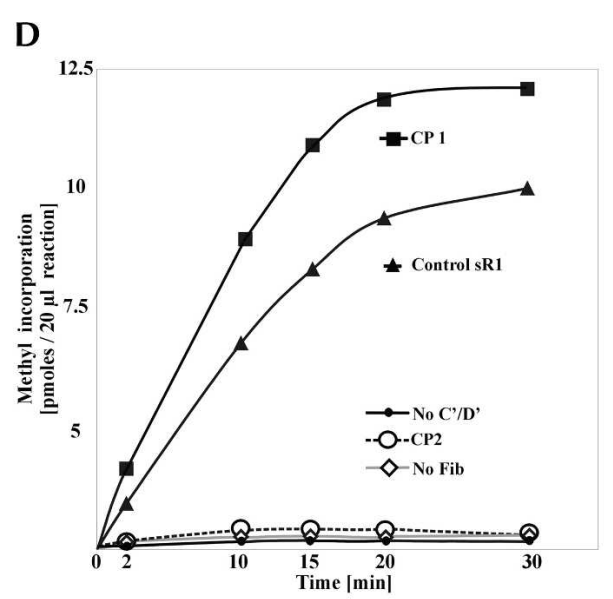

E

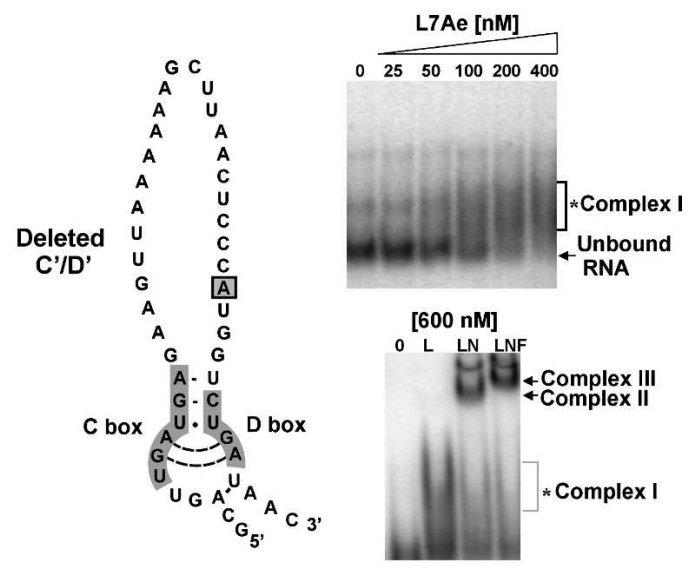

F
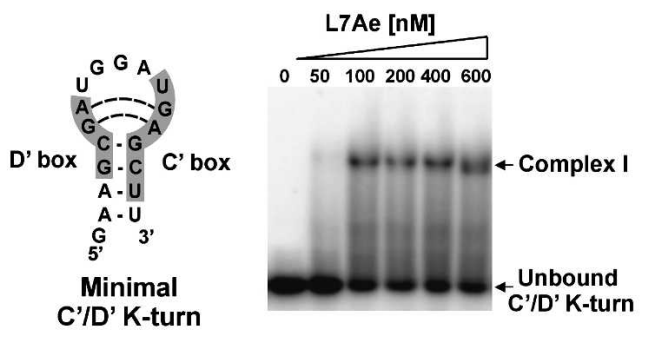

$\underset{\mathrm{L}}{[600 \mathrm{nM} \text { LNF }}$

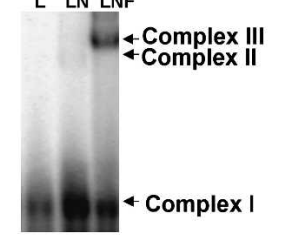

FIGURE 3. (Legend on next page) 
minimal RNA, there was virtually no Complex II formation upon addition of L7Ae and Nop5 (see LN lane in Fig. 3F). However, upon the addition of Fib, full assembly of a particle occurs (see LNF lane in Fig. 3F). This suggests that the interaction of Fib with Nop5 causes a structural rearrangement within the Nop5 protein that allows it to interact more efficiently with the $\mathrm{C}^{\prime} / \mathrm{D}^{\prime}$ truncated RNA substrate. The complex could not be tested for methylation activity because it lacks an associated guide sequence.

\section{Multiple and single nucleotide substitutions in the $C^{\prime} / D^{\prime}$ motifs}

The functional role that the K-turn formed by the $\mathrm{C}^{\prime} / \mathrm{D}^{\prime}$ box elements plays during the methyl transfer reaction has been further investigated by partially or entirely replacing the nucleotide sequence of the $\mathrm{C}^{\prime}$ - and/or $\mathrm{D}^{\prime}$-boxes by a poly $(\mathrm{C})$ track. The protein-binding abilities of these mutant RNAs were assayed in gel retardation experiments in the presence of the L7Ae, Nop5, and Fib core proteins. It should be noted that the gel shift assay reflects global binding affinity of the proteins to the respective guide sRNAs, all of which contain one altered and one native K-turn motif; the stoichiometry of binding was not determined. All mutants tested displayed qualitative L7Ae-Nop5 and L7Ae-Nop5-Fib binding properties indistinguishable from the wild-type sR1 RNA, although some heterogeneity was observed in the reactions containing only the L7Ae protein (i.e., relative mobility and amount of complex; see above). In spite of the lack of apparent differences in the assembly properties of higher-order complexes, the particles containing the mutant sRNAs exhibited different levels of methylation activity. Substitution of the first half of the $\mathrm{C}^{\prime}$-box and of the nucleotides separating the $\mathrm{C}^{\prime}$ from the $\mathrm{D}^{\prime}$ element by poly $(\mathrm{C})$ (Fig. 2F, nucleotide positions 27-33) resulted in a complex that retained $\sim 50 \%$ of the control methylation activity, whereas a mutant containing a poly $(\mathrm{C})$ track in the $\mathrm{D}^{\prime}$-box (Fig. $2 \mathrm{G}$ ) retained only $\sim 35 \%$ of the activity. The simultaneous alteration of $\mathrm{C}^{\prime}$ and $\mathrm{D}^{\prime}$ motifs (Fig. 2H) had a cumulative negative effect and lowered the activity to $\sim 17 \%$ of the control value.

Two of the predominant features that contribute to the K-turn stability and mediate interaction with the L7Ae protein are the presence of two non-Watson-Crick G:AA:G base pairs and an unpaired $U$ residue that protrudes out of the RNA helix. To probe the importance of these features, we introduced into separate sRNAs a G32C substitution that disrupts the second G:A base pair and a U31A substitution that replaces the conserved protruding $U$ with A (Fig. 2I,J). The first substitution reduced the activity of the complex to $\sim 20 \%$ of the wild-type control and was roughly equivalent to replacing the entire $\mathrm{C}^{\prime} / \mathrm{D}^{\prime}$ region with poly $(\mathrm{C})$. In contrast, the U31A replacement had a less prominent effect on the methylation activity of the complex. Finally, we constructed an sRNA that is entirely deleted for the $\mathrm{D}^{\prime}$ and $\mathrm{C}^{\prime}$ regions (Figs. $2 \mathrm{~K}, 3 \mathrm{E}$ ). Although this sRNA retains the guide sequence adjacent to the D-box and is able to form Complexes II and III, it exhibits virtually no methylation activity (Fig. 3D).

\section{Nucleotide substitutions in stem II of the $C / D$ and $C^{\prime} / D^{\prime}$ K-turn motifs}

In the majority of methylation guide RNAs present in Eukarya and Archaea, a noncanonical U-U pair is generally conserved in C/D-box K-turns at the top of stem II, immediately adjacent to the first of the two sheared G:AA:G base pairs. In eukaryotes, cross-linking data point to a direct role of the $\mathrm{U}-\mathrm{U}$ pair in the interaction with Nop56 or Nop58, and substitution by a G-C pair prevents assembly of the resulting mutant with Nop56/58, as assayed by immunoprecipitation (Cahill et al. 2002; Watkins et al.

FIGURE 3. RNA-protein gel shift and methylation activity assays. On the left side of each panel, the secondary structure models of wild-type and mutant sR1s are depicted. The positions of C-, D'-, C'-, and D-boxes are indicated, and the two sheared G:A base pairs (dashed line) in each Kturn are highlighted. The A residue that base-pairs with the U52 methylation site in the target rRNA is boxed. For the wild-type sR1 illustration, the target sequence is shown (gray) base-paired to the guide. This interaction is expected to occur in the same way between the guides and target in all of the other mutant sRNAs but is not depicted. Shown on the right side of each panel is stepwise complex formation monitored by gel shift assays using a uniformly labeled sR1 RNA (wild type or mutant) and L7Ae protein, (top autoradiogram; Complex I) or in the bottom autoradiogram, L7Ae (L), L7Ae, and Nop5 (LN; Complex II), or L7Ae, Nop5, Fib (LNF; Complex III). It should be noted that the gel shift assays are a qualitative measure of complex formation and do not provide information on the protein stoichiometry in the particles even though the migration profiles especially for Complexes II and III are remarkably similar. Occasionally, bands with mobility slower than Complex III are observed; these appear to be higher-order aggregates of unknown composition. (A) (Wt) Wild-type sR1; (B) (CP1) a circular permutation of sR1 where the normal $5^{\prime}$ and $3^{\prime}$ ends of the RNA are joined by ligation between nucleotides A2 and U54, new $3^{\prime}$ and $5^{\prime}$ ends are introduced between nucleotides $\mathrm{U} 27$ and A28 and a G(28)A mutation was introduced to provide a base pair to close the terminal loop; (C) CP2, a circular permutation of sR 1 where the normal $5^{\prime}$ and $3^{\prime}$ ends of the RNA are joined by ligation between nucleotides A2 and U54 and new $3^{\prime}$ and $5^{\prime}$ ends are introduced between nucleotides G14 and U15; $(E)$ (deleted $\mathrm{C}^{\prime} / \mathrm{D}^{\prime}$ ) a derivative of sR1 in which the nucleotides between positions 24 and 34 are deleted to remove the K-turn formed by the $\mathrm{D}^{\prime}$ - and $\mathrm{C}^{\prime}$-box sequences [ ${ }^{*}$ Complex I is more heterogeneous than with the other mutant sRNAs, whereas Complexes II and III are efficiently formed]; $(F)$ (mini C'/D') fragment corresponding to nt positions 21-37 of wild-type sR1; $(D)$ methylation activities were determined as described in Materials and Methods; each reaction contained 120 pmol of sR1 (wild type or mutant), $120 \mathrm{pmol}$ of RNA target, $4 \mathrm{pmol}$ of each protein, and $60 \mathrm{pmol}$ of $\left[{ }^{3} \mathrm{H}\right.$-methyl]SAM. In the control reaction, fibrillarin protein was omitted. Individual $20-\mu \mathrm{L}$ reactions were removed at 2, 10, 15, 20, and $30 \mathrm{~min}$ and precipitated 15 min on ice after addition of $5 \%$ TCA. Precipitates were collected on filters and dried, and the incorporation of ${ }^{3} \mathrm{H}$ radioactivity was measured by scintillation counting. 
2002). In wild-type sR1, stem II of the C/D-box K-turn has this U-U base pair, whereas the $\mathrm{C}^{\prime} / \mathrm{D}^{\prime}$ stem II has a G$\mathrm{C}$ base pair at this position (Fig. 1B,C). To probe the importance of this U-U pair, we constructed two different mutants of sR1. In the first mutant, the $C-G$ base pair present at this position in stem II of the $\mathrm{C}^{\prime} / \mathrm{D}^{\prime} \mathrm{K}$-turn motif was mutated to $\mathrm{U}-\mathrm{U}$ and the $\mathrm{D}$-box guide was repositioned adjacent to the $\mathrm{D}^{\prime}$-box (Fig. 2L). This mutant sRNA was able to assemble into an RNP complex in the presence of L7Ae, Nop5, and Fib, as judged in the standard gel mobility shift assay, but surprisingly was virtually devoid of methylation activity ( $6 \%$ of the sR 1 control). In the second mutant, the sequence of stem II of the C/D K-turn was replaced with a $\mathrm{C}^{\prime} / \mathrm{D}^{\prime}$-like sequence resulting in a $\mathrm{G}-\mathrm{C}$ rather than a $\mathrm{U}-\mathrm{U}$ base pair at the critical position (Fig. 2M). This RNA was efficiently assembled into an RNP complex, and the complex exhibited methylation activity that was essentially identical to the wild-type sR1 control (96\% initial methyl incorporation rate and 82\% maximal incorporation). We concluded from these results that the presence of the conserved $U-U$ pair in the terminal $C / D$ K-turn of sR1 RNA is not essential for activity and that substitution by a canonical G-C base pair is equally well tolerated. Conversely, replacement of the Watson Crick G-C pair that closes the $\mathrm{C}^{\prime} / \mathrm{D}^{\prime}$ internal K-turn by a U-U interaction does not provide methylation function to the inactive internal $\mathrm{C}^{\prime} / \mathrm{D}^{\prime}$ motif.

\section{Replacements of C/D- and $C^{\prime} / D^{\prime}$-box K-turns}

Five mutants with alterations in the K-turns formed by the $\mathrm{C} / \mathrm{D}$ - and $\mathrm{C}^{\prime} / \mathrm{D}^{\prime}$-box regions were constructed and analyzed. The first had the entire $\mathrm{C}$ - and D-box sequences replaced by poly $(\mathrm{C})$ tracks (Fig. $2 \mathrm{~N}$ ). This mutant RNA was able to bind L7Ae, Nop5, and Fib but was totally devoid of methylation activity (5.5\% incorporation rate).

The K-turn 38 in the $23 \mathrm{~S}$ rRNA of the Haloarcula marismortui large ribosome subunit crystal structure is stable and does not associate with ribosomal proteins (Klein et al. 2001). Further characterization of this unusual K-turn motif demonstrated that it requires only the presence of low levels $(10 \mathrm{mM})$ of $\mathrm{Mg}^{2+}$ to form a stable, selffolding RNA structure (Matsumura et al. 2003). Other K-turns typically require the presence of higher $\mathrm{Mg}^{2+}$ concentrations and assistance by proteins for correct folding. Based on the K-turn 38 unique stability and on the recently proposed chaperone function for L7Ae for facilitating K-turn formation in C/D-box RNAs, we constructed three sR1 derivatives. In one, the C/D K-turn was replaced with K-turn 38; in the second, the $\mathrm{C}^{\prime} / \mathrm{D}^{\prime} \mathrm{K}$-turn was replaced with K-turn 38; and in the third, both $\mathrm{C} / \mathrm{D}$ and $\mathrm{C}^{\prime} / \mathrm{D}^{\prime} \mathrm{K}$ turns were replaced with K-turn 38 (Figs. 2O, 4A; data not shown). Complex formation was detected with all three RNAs using standard band shift assays in the presence of all three proteins, and a $1 \mathrm{mM} \mathrm{Mg}^{2+}$ concentration (Fig. 4B). In contrast, complexes between the sRNAs and the Nop5 and Fib proteins in the absence of L7Ae could not be detected by standard gel retardation assays or by filter binding assays even when the $\mathrm{Mg}^{2+}$ concentration was increased to $10 \mathrm{mM}$ (data not shown). This implies that the binding of L7Ae protein is required for binding of Nop5 and Fib even when the K-turn structure is stably folded. We tested these mixtures for in vitro methylation activity. None of the K-turn-38-containing mutant sRNAs (either in the presence or the absence of L7Ae protein or at 1 or $10 \mathrm{mM} \mathrm{Mg}^{2+}$ concentrations) was efficient in guiding methyl transfer to the RNA target (Fig. 4C; data not shown). Together, these results indicate that a stabilized K-turn 38 cannot replace the typical C/D or $C^{\prime} / D^{\prime} \mathrm{K}$-turns in sR1 and that the L7Ae protein is required for the assembly (and methylation activity) of the complex.

In the fifth mutant of this group, the C/D K-turn motif was replaced by the $S$. solfataricus sequence corresponding to K-turn 15, initially identified as the binding site for the L7Ae protein in the crystal structure of the $50 \mathrm{~S}$ ribosome subunit from H. marismortui (Klein et al. 2001). Although the overall structure of K-turn 15 is unusual in that it contains an A:U:A (A:U:G in H. marismortui) base triple in place of one of the two sheared G:A-A:G base pairs (see Figs. 2P, 4A), the motif efficiently binds the L7Ae protein (Fig. 4B), and in the context of sR1, the complex formed in the presence of L7Ae, Nop5, and Fib is at least as active as wild-type sR1 in the methylation reaction (Figs. 2P, 4C). Surprisingly, the methylation activity of the K-turn-15containing RNP complex was, unlike the wild-type sR1containing complexes, sensitive to $\mathrm{Mg}^{2+}$ concentration.

\section{DISCUSSION}

In an attempt to define better the relationship that exists between the structure of a typical archaeal methylation guide RNA and its methylation function, we constructed an array of rearrangements and nucleotide substitution mutations in the well-characterized $S$. acidocaldarius model sR1 C/D-box RNA. By assaying each mutant guide RNA for the ability to assemble with L7Ae, Nop5, and Fib into ribonucleoprotein complexes and the capacity, when assembled as an RNP, to function in the methyl transfer reaction, we showed that the RNA-protein interactions involved in assembly with L7Ae, Nop5, and Fib are much less sensitive to mutational perturbations of the sRNA than is the methylation activity of the resulting complex. We have also shown that the presence of K-turn 15 (the binding site of the L7Ae protein in the $50 \mathrm{~S}$ ribosomal subunit) can fully replace the $C / D$ motif in $s R 1$, whereas the presence of the stabilized K-turn 38 in either the $\mathrm{C} / \mathrm{D}$ or $\mathrm{C}^{\prime} / \mathrm{D}^{\prime}$ position renders the guide complex inactive in the methylation reaction. This is surprising since the structure of K-turn 15 is highly unusual in that an $\mathrm{A}: \mathrm{U}: \mathrm{A}$ base triple replaces one 

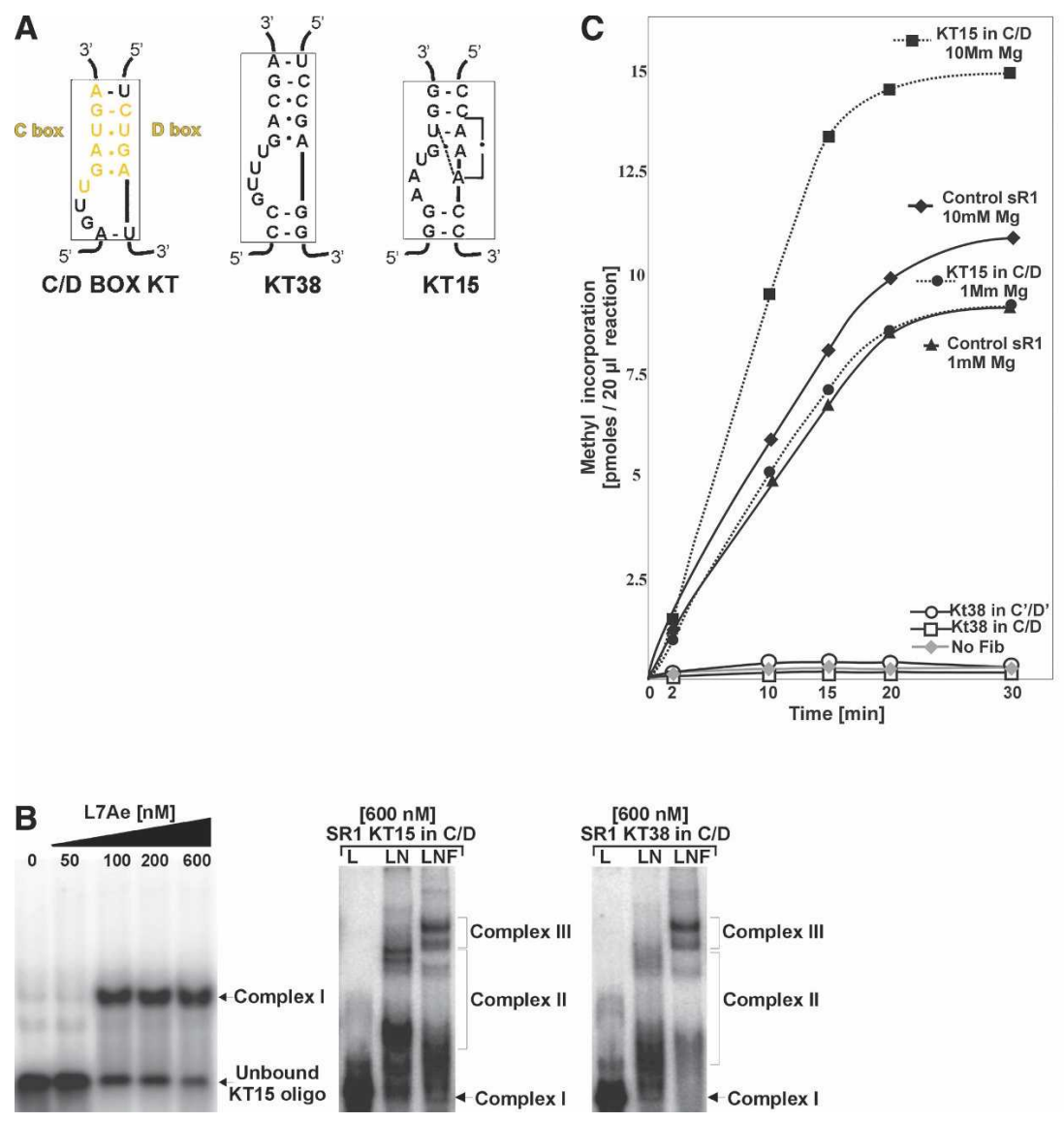

FIGURE 4. The activity of chimeric sRNAs containing K-turn 38 and K-turn 15 derived from $23 \mathrm{~S}$ rRNA. Chimeric sR1 sRNAs were constructed that contained the K-turn 38 sequence form H. marismortui $23 \mathrm{~S}$ rRNA or the K-turn 15 sequence from S. solfataricus $23 \mathrm{~S}$ rRNA. (A) The sequence and secondary structure of wild-type and mutant $\mathrm{K}$-turns used in this study are illustrated: C/D-box (left), K-turn 38 in C/D-box (center), K-turn 15 in C/D-box (right). (B) The gel shift analysis using an isolated K-turn 15 containing oligonucleotide (left panel), the chimeric sRNA containing the K-turn 15 in the C/D-box position (center), and the chimeric sRNA containing the K-turn 38 in the C/D-box position (right panel) is illustrated. $(C)$ The complexes were assayed for methyl incorporation using the standard reaction conditions. The composition and conditions for the reaction are as described in Materials and Methods and in the legend to Figure 3D, with the modification that a high $\mathrm{Mg}^{2+}$ concentration $(10 \mathrm{mM})$ was in some cases present in the binding buffer.

of the two sheared G:A base pairs in the canonical C/D motif, whereas K-turn 38 appears to resemble more closely the canonical K-turn structure (Fig. 4A).

\section{Circular permutations of sR1}

Mutagenesis by circular permutation is a powerful tool for investigating the role of functionally relevant domains in the folded tertiary structure of RNA. In Tetrachimena group I and RNase P ribozymes, systematic mutagenesis by circular permutation has revealed the regions critical for correct folding and activity of these highly structured RNAs. Moreover, these studies reveled a direct correlation between the topology and proximity of RNA domains in the primary sequence and the kinetics and thermodynamics of RNA folding (Pan et al. 1999; Woodson 2002). In our study, the positioning through circular permutation of the sR1 $5^{\prime}$ - and $3^{\prime}$-termini between the $\mathrm{C}^{\prime}$ - and $\mathrm{D}^{\prime}$-boxes was found to stimulate methylation activity (CP1A mutant) (Figs. $2 \mathrm{~B}, 3 \mathrm{~B}, \mathrm{D})$ whereas the positioning of the ends between the C- and $\mathrm{D}^{\prime}$-boxes completely abolished methylation function (CP2 mutant) (Figs. 2C, 3C,D). These results indicate first that in sR1, the $C / D$ and $C^{\prime} / D^{\prime}$ motifs could form functional K-turns in the absence of a terminal stem (canonical stem I), and second, that the methylation guide region in sR1 can be coupled to either a terminal or an internal K-turn structure. In contrast, when connectivity was disrupted by positioning the $5^{\prime}$ - and $3^{\prime}$ ends between the $\mathrm{D}^{\prime}$ - and C-boxes as in the $\mathrm{CP} 2$ construct, methylation activity was abolished (Fig. 2C). The importance of rigid connectivity in the linkers between the C- and $\mathrm{D}^{\prime}$ - and the $\mathrm{D}^{\prime}$ - and C-boxes has recently been demonstrated. The optimal separation of Cand $\mathrm{D}^{\prime}$ - and the $\mathrm{D}^{\prime}$ - and C-boxes in archaeal sRNAs was shown to be tightly clustered around a length of $12 \mathrm{nt}$ (Tran et al. 2005); symmetrical and asymmetrical increases or decreases in this optimal length resulted in a decrease in the methylation activity of the RNP complexes.

\section{Relocation of the guide sequence from the $D$ to the $D^{\prime}$ position}

Relocation of the D-box guide adjacent to the $\mathrm{D}^{\prime}$-box either in the native sR1 or in the CP1A mutant sRNA resulted in more than sixfold reduction of the methylation activity (Fig. 2D,E). We initially interpreted this as being the result of sequence or structural deviation of the $\mathrm{C}^{\prime}$ and $\mathrm{D}^{\prime}$ elements relative to the $\mathrm{C}$ and $\mathrm{D}$ consensus. However, we found that an sR1mutant containing two $\mathrm{C}^{\prime} / \mathrm{D}^{\prime}$ motifs (one replacing the canonical $\mathrm{C} / \mathrm{D}$ motifs in the terminal position) retains $>80 \%$ of the activity exhibited by the sR1 control (Fig. 2M). We noticed further in the predicted secondary structure of the GR and CP1B mutants (Fig. 2D,E) that repositioning of the antisense guide sequence adjacent to the $\mathrm{D}^{\prime}$-box reduced the stability of the flanking stem II, from four Watson-Crick base pairs in the wild type to three and two, respectively. In contrast, in the $\mathrm{C}^{\prime} / \mathrm{D}^{\prime}-\mathrm{C}^{\prime} / \mathrm{D}^{\prime}$ mutant, the stability of the stem II flanking the internal $\mathrm{C}^{\prime} / \mathrm{D}^{\prime}$ motif is 
unaffected, and this correlates with the preserved activity of this mutant (Fig. 2M). Moreover, a mutant carrying the guide relocated by the $\mathrm{D}^{\prime}$-box and a U-U substitution in the first $\mathrm{W}-\mathrm{C}$ base pair, at the top of internal $\mathrm{C}^{\prime} / \mathrm{D}^{\prime}$ stem II, appears completely inactive in the methylation reaction (Fig. 2L). Taken together, these results demonstrate that in sR1, the internal $\mathrm{C}^{\prime} / \mathrm{D}^{\prime}$ element cannot direct target methylation and that the stem II adjacent to this motif plays an essential structural role in the function of the RNP, possibly by promoting correct packing of the RNA during the early folding or assembly events. In support of this observation, Szewczak et al. (2005) have noted that the $C^{\prime} /$ $\mathrm{D}^{\prime}$ motif of a standard eukaryotic methylation guide that typically does not interact with the $15.5-\mathrm{kDa}$ protein could bind the protein if a terminal 4-bp stem is introduced by circular permutation between the $\mathrm{C}^{\prime}$ and $\mathrm{D}^{\prime}$ elements.

\section{Multiple and single nucleotide substitutions in the $C^{\prime} / D^{\prime}$ motif}

We introduced nucleotide substitutions to assess the importance of the tandem G:A-A:G interactions and of the protruding $\mathrm{U} 31$ residue for the function of the D-boxassociated guide. Substitution by poly $(\mathrm{C})$ tracks in the first half of the $\mathrm{C}^{\prime}$-box, or in the entire $\mathrm{D}^{\prime}$-box and a combination of both, reduces $\mathrm{D}$ guide methylation activity to onehalf, one-third, and less than one-fifth, respectively, when compared to the sR1 control (Fig. 2F-H). Earlier mutational and structural studies have established the importance of the adjacent noncanonical G:A-A:G interaction. Point mutations that disrupt one of the G:A shared pairs and presumably prevent K-turn formation, reduce or abolish the methylation function of both the disrupted and intact box C/D motifs (Bortolin et al. 2003; Rashid et al. 2003; Tran et al. 2003; Moore et al. 2004). However, in contrast with previous results published by Tran et al. (2003) that define the C/D-box element as being the minimal methylation competent guide structure, we find that for $S$. acidocaldarius $\mathrm{sR} 1$, deletion of the $\mathrm{C}^{\prime} / \mathrm{D}^{\prime}$ motif completely prevents methylation, but not the assembly function of the C/D motif (Figs. 2K, 3E). Moreover, our results suggest that the stability of stem II (that normally seals the $C^{\prime} / D^{\prime}$ K-turn structure) modulates the activity of the D-boxassociated guide (see above). As shown in Figure 2, F, G, and $\mathrm{H}$, the gradual loss of activity with the depicted mutants correlates with a gradual reduction in the stability of stem II from five, to four, to three (interrupted by a C:C mismatch) Watson-Crick base pairs. This could also explain the more pronounced effect of the G32C point mutation when compared to the C-triplet substitution in the first half of the $\mathrm{C}^{\prime}$-box and to the U31A point mutation. Again, activity correlates with the number of canonical base pairs that form an uninterrupted helix (Fig. $2 \mathrm{~F}, \mathrm{I}, \mathrm{J})$. Taken together, these findings confirm the cooperative role of the internal $\mathrm{C}^{\prime} / \mathrm{D}^{\prime}$ and terminal $\mathrm{C} / \mathrm{D} \mathrm{K}$ - turns and suggest a more prominent connection between the stability of the $\mathrm{C}^{\prime} / \mathrm{D}^{\prime}$ stem II and the $\mathrm{D}$ guide function than was previously imagined.

A recent study of the sequence connecting the $\mathrm{C}^{\prime}$ - and $\mathrm{D}^{\prime}$-box sequences of Pyrococcus sRNAs suggests that this terminal loop has an optimal length of $3 \mathrm{nt}$ with a consensus of RNK (where R is A or G; $\mathrm{N}$ is any nucleotide; and $\mathrm{K}$ is $\mathrm{G}$, U, or A) (Nolivos et al. 2005). The terminal K-loop in the $S$. acidocaldarius sR1 deviates from the pyrococcal consensus; it is $4 \mathrm{nt}$ in length and has a UGGA sequence. We found that mutation of this loop sequence in $\mathrm{sR} 1$ to $\operatorname{poly}(\mathrm{C})$ (along with disruption of the sheared A:G base pairs) (Fig. 2F) had only a modest twofold effect on the methylation activity of the complex. Moreover, we noted that the CP1A circular permutation construct also has a 4-nt terminal loop between the D- and C-boxes (UAGU) and functions better than wild-type $\mathrm{sR} 1$ in the methylation reaction (Fig. 2B).

\section{Nucleotide substitutions in stem II of the K-turn motifs}

Replacement of the $C / D$ motif with a $C^{\prime} / D^{\prime}$ motif has a minimum effect on the methylation activity. In contrast, introduction of a U-U base pair at the top of stem II in the $\mathrm{C}^{\prime} / \mathrm{D}^{\prime} \mathrm{K}$-turn does not confer methylation activity to the already inactive $C^{\prime} / D^{\prime}$ motif (Fig. 2L,M). These findings demonstrate two important structural features of sR1 methylation guide. First, the U-U pair closing stem II of the C/D K-turn that is conserved in eukaryotes is not essential in the $S$. acidocaldarius sR1 RNA; the substitution by a G-C canonical pair is equally tolerated (Fig. 2M), consistent with structural data showing that replacement of $\mathrm{U}-\mathrm{U}$ by $\mathrm{G}-\mathrm{C}$ at this position causes only a minor change in the bending angle of the RNA backbone, without altering the binding affinity of the L7Ae protein (Moore et al. 2004). In contrast to the stable stem II associated with the nonguiding $\mathrm{C}^{\prime} / \mathrm{D}^{\prime} \mathrm{K}$-turn (see above), a weakened or less stable stem II in the C/D K-turn associated with the guide function is required for activity. Second, the replacement of the G-C Watson-Crick base pair by a U-U pair at the corresponding location in the $\mathrm{C}^{\prime} / \mathrm{D}^{\prime} \mathrm{K}$-turn does not promote target methylation from the $\mathrm{D}^{\prime}$-box (Fig. 2L).

\section{sRNAs with nonstandard K-turns}

The importance of intact K-turns was demonstrated by the lack of activity of mutants in which both $\mathrm{C}$ and $\mathrm{D}$ or $\mathrm{C}^{\prime}$ and $\mathrm{D}^{\prime}$ sequences were replaced by poly $(\mathrm{C})$ tracks (Fig. $2 \mathrm{H}, \mathrm{N}$ ). To define better the specific features of the $\mathrm{C} / \mathrm{D}$ and $\mathrm{C}^{\prime} / \mathrm{D}^{\prime}$ $\mathrm{K}$-turns and to assess the proposed chaperone function of the L7Ae protein, we first replaced, one at the time or together, the C/D and $C^{\prime} / D^{\prime}$ K-turn motifs in sR1 with the sequence corresponding to K-turn 38 or K-turn 15 identified in the $23 \mathrm{~S}$ rRNA of $H$. marismortui. In the crystal 
structure of the large ribosome subunit, there are no proteins associated with K-turn 38, whereas the multifunctional L7Ae protein is associated with K-turn 15.

The K-turn 38 sequence is unusual in that it is able to fold into a compact K-turn structure in the presence of $10 \mathrm{mM}$ $\mathrm{Mg}^{2+}$ without the aid of a protein chaperone. The three mutant sRNAs that we constructed, containing the K-turn 38 motif in the $\mathrm{C} / \mathrm{D}, \mathrm{C}^{\prime} / \mathrm{D}^{\prime}$ or $\mathrm{C} / \mathrm{D}$ and $\mathrm{C}^{\prime} / \mathrm{D}^{\prime}$ motifs, were unable to associate with Nop5 and Fib in the absence of the L7Ae protein at either 1 or $10 \mathrm{mM} \mathrm{Mg}^{2+}$, and none of these mixtures was active in the methylation reaction. This result implies that the L7Ae protein acts not only as an RNA chaperone to mediate the folding of C/D-box sRNA Kturns, but is also actively involved in nucleating the addition (possibly through direct protein-protein interactions) of the Nop5 and Fib proteins to these complexes. We also tested the assembly and methylation activity of the three K-turn-38containing sRNAs in the presence of L7Ae (Fig. 4B; data not shown); all three RNAs form higher-order complexes in the presence of the three proteins, but surprisingly none of the complexes were active in methylation. We do not understand what features of K-turn 38 make it incompatible with C/Dbox sRNA function, although we noted (1) the unusual C:C mismatch adjacent to the G:A-A:G pairs, at the base of stem II; (2) the presence of an additional nucleotide (four rather than three) in the asymmetric loop; and (3) the inherent rigidity and inflexibility of the structure.

\section{Concluding remarks}

In summary, we have shown that in the $S$. acidocaldarius sR1 single guide sRNA, the C/D and $\mathrm{C}^{\prime} / \mathrm{D}^{\prime} \mathrm{K}$-turns play distinct roles in the methylation reaction (Fig. 5). As summarized in Figure 5, the C/D K-turn is associated with the methyl transfer function and is active when located either at the terminal or internal position. The internal $C^{\prime} / D^{\prime}$ K-turn appears not to participate directly in the methyl transfer reaction; instead, it seems to play a structural role by facilitating the correct folding of the RNA guide. We observed that the stable stem II adjacent to the $C^{\prime} / D^{\prime}$ element is required for full methylation activity guided by the opposite $\mathrm{C} / \mathrm{D}$ element.

Many crenarchaeal methylation guide RNAs, unlike the eukaryotic and euryarchaeal guide sRNAs, lack the ubiquitous terminal stem structure that closes the loop at the top of the K-turn formed by the C- and D-box sequences. Similar to sR1, many of the $S$. acidocaldarius sRNAs (like sR4 and sR7, accession numbers AF195098 and AF195101, respectively) contain a stable $3-5$-bp stem II associated with the internal K-turn formed by the $\mathrm{C}^{\prime}$ and $\mathrm{D}^{\prime}$ motifs. We suggest that the stability of this structure compensates for the lack of the terminal helix and that this is an important element required for the correct folding of the guide RNA.

The demonstration that chimeric guide RNAs containing one or both of the K-turns replaced by the self-folding

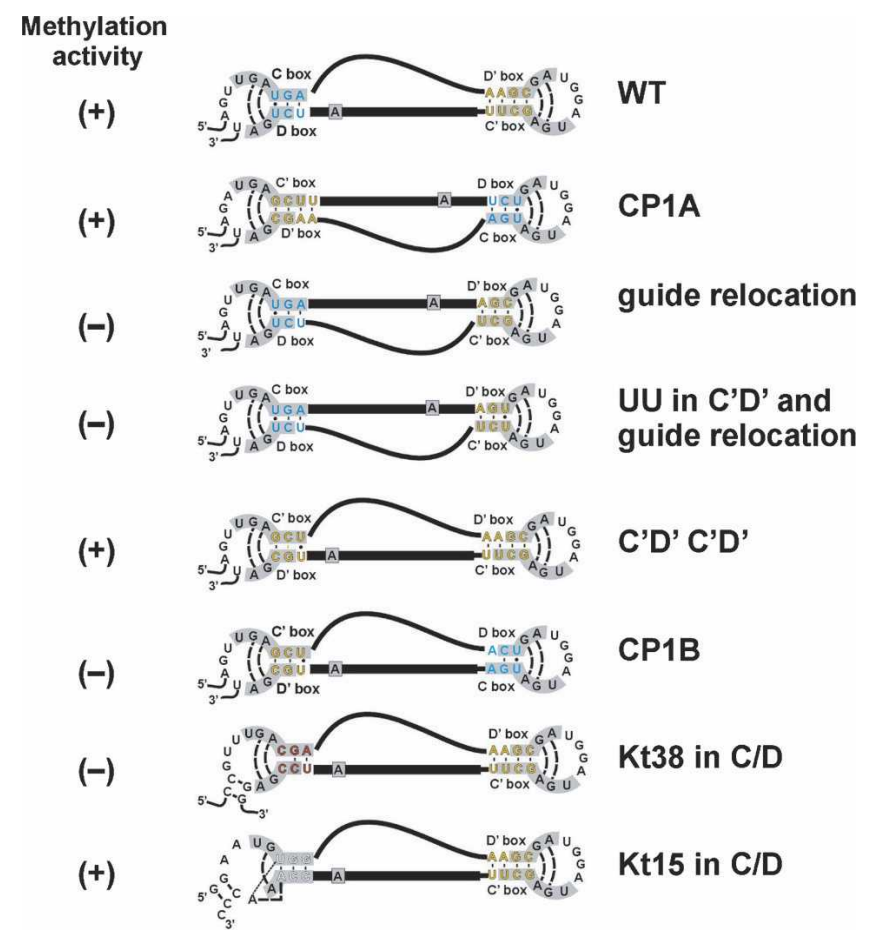

FIGURE 5. The stability of the stem II of the $\mathrm{C}^{\prime} \mathrm{D}^{\prime} \mathrm{K}$-turn correlates with the methylation activity of mutant sRNAs. The sequences of the stem IIs flanking the C/D (blue), C'/D' (yellow), K-turn 38 (red), or K-turn 15 (white) motifs are shown. The activities of the various sRNAs in the in vitro methylation reaction are indicated on the left: $(+)$ indicates $>80 \%$ and $(-)$ indicates $<15 \%$ methylation activity when compared to the sR1 sRNA control.

K-turn 38 are inactive in methyl transfer substantiates the idea that L7Ae, in addition to acting as a chaperone for the formation of the RNA K-turn motifs, plays a more prevalent role in the reaction, possibly by mediating RNAprotein and protein-protein interactions within the dynamic RNP complex. In contrast to K-turn-38-containing sRNAs chimeras, a chimeric sR1 guide containing the unusual K-turn 15 is fully functional in the guided ribose methylation reaction. This expands the repertoire of documented features associated with the guided ribose methylation function in Sulfolobus and raises the prospect that other noncanonical C/D-box (K-turn) containing sRNAs that were recently identified in functional screens (and shown to interact efficiently with L7Ae, Nop5, and Fib) may possess bona fide methylation function.

\section{MATERIALS AND METHODS}

\section{Oligonucleotides}

All oligonucleotides were synthesized by Operon Biotechnology Inc. The sequence of the oligonucleotide pairs used to generate the DNA templates for the target RNA and each of the mutants used in this study are listed below. All the DNA templates contained the 
T7 promoter region for the T7 RNA polymerase at their $5^{\prime}$-end (underlined).

rRNA target

AO63.1: 5' -GTAATACGACTCACTATAGGGATAAGCCATGGG AG-3'

AO65: 5' -TATTTAGGTGACACTATAGGTTAGCCACGTGTTAC TCAGCC-3'

CP1A mutant

AO98: 5'-GTAATACGACTCACTATAGACAGATGAGCTTAACT CCCATGGTCTGATAGTTGATG-3'

AO99: 5' -AATCGCTTTTTTAACTTCTCATCAACTATCAG-3'

CP2 mutant

AO107: 5' -GTAATACGACTCACTATAGTAAAAAAGCGATGGA TGAGCTTAACTCCCATGGTCT-3'

AO108: 5' -CTTCTCATCAACTATCAGACCATGGGAGTTAAGC TC-3'

GR mutant

OHE116: 5'-GTAATACGACTCACTATAGCAGTTGATGATAAC TCCCATGGAGCGATGGATGAGCT-3'

OHE117: 5'-GTTATCAGATTTTTTAACTTCAGCTCATCCATC GCTCC-3'

CP1B mutant

OHE114: 5'-GTAATACGACTCACTATAGAGATGAGCTGAAG TTAAAAAACTGATGGTTGATGA-3'

OHE115: 5'-GTTATCGCACCATGGGAGTTATCATCAACCATC AGTTTTTT-3'

${ }^{*} C^{\prime} \operatorname{poly}(C)$ mutant

OHE77: 5'-GTAATACGACTCACTATAGCAGTTGATGAGAAG TTAAAAAAGCGACCCCCCCGCT-3'

OHE78: 5'-GTTATCAGACCATGGGAGTTAAGCGGGGGGGTC GCTTT-3'

$D^{\prime} \operatorname{poly}(C)$ mutant

OHE75: 5'-GTAATACGACTCACTATAGCAGTTGATGAGAAG TTAAAAAACCCCTGGATGAGCT-3'

OHE76: 5'-GTTATCAGACCATGGGAGTTAAGCTCATCCAGGGG TTT-3'

$C^{\prime} / D^{\prime} \operatorname{poly}(C)$ mutant

OHE61: 5'-GTAATACGACTCACTATAGCAGTTGATGAGAAG TTAAAAAACCCCCCCCCCCGCT-3'

OHE62: 5'-GTTATCAGACCATGGGAGTTAAGCGGGGGGGGG GGTTT-3'
G32C mutant

OHE100: 5' -GTAATACGACTCACTATAGCAGTTGATGAGAAG TTAAAAAAGCGATGGATCAGCT-3'

OHE101: 5'-GTTATCAGACCATGGGAGTTAAGCTGATCCATCG CTTT-3'

\section{U31A mutant}

OHE79: 5'-GTAATACGACTCACTATAGCAGTTGATGAGAAG TTAAAAAAGCGATGGAAGAGCT-3'

OHE80: 5'-GTTATCAGACCATGGGAGTTAAGCTCTTCCATC GCTTT-3'

$\cup U$ in $C^{\prime} / D^{\prime}$ mutant

OHE120: 5'-GTAATACGACTCACTATAGCAGTTGATGATAAC TCCCATGGAGTGATGGATGATCTG-3'

OHE121: 5'-GTTATCAGATTTTTAACTTCAGATCATCCATCA CTCC-3'

Double $C^{\prime} / D^{\prime}$ mutant

OHE118: 5' -GTAATACGACTCACTATAGCAGTTGAGCTGAAG TTAAAAAAGCGATGGATGAGCT-3'

OHE119: 5'-GTTATCGCACCATGGGAGTTAAGCTCATCCATC GCTTTTTT-3'

$C / D \operatorname{poly}(C)$ mutant

OHE73: 5 '-GTAATACGACTCACTATAGCAGTCCCCCCGAAG TTAAAAAAGCTT-3'

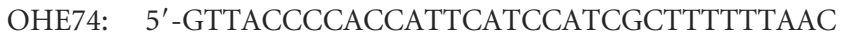
TTC-3'

C/D KT-38 mutant

OHE108: 5' -GTAATACGACTCACTATAGCCGTTTGACGAGAA GTTAAAAAAGCGATGGATGAGCT-3'

OHE109: 5'-GTCCTCGGACCATGGGAGTTAAGCTCATCCAT CGCTTTTTT-3'

$C^{\prime} / D^{\prime} K T-38$ mutant

OHE110: 5' -GTAATACGACTCACTATAGCAGTTGATGAGAAG TTAAAAAACCGAGCGTTTGACG-3'

OHE111: 5'-GTTATCAGACCATGGGAGTTAACGTCAAACGC TCGGTTTTTTA-3'

C/D KT-15 mutant

OHE130: 5' -GTAATACGACTCACTATAGGGAATGTGGTGCAG TTAAAAAAGCGATGGATGAGCT-3' 


\section{OHE131: 5'-GGGTTTGGACCATGGGAGTTAAGCTCATCCAT CGCTTT-3'}

$C / D C^{\prime} / D^{\prime} K T-38$ mutant

OHE138: 5' -GTAATACGACTCACTATAGCCGTTTGACGAGAA GTTAAAAAACCGAGCGTTTGACG-3'

OHE139: 5'-GTCCTCGGACCATGGGAGTTAACGTCAAACGC TCGGTTTTTTA-3'

\section{Deleted $C^{\prime} / D^{\prime}$ mutant \\ OHE53: 5 '-GTAATACGACTCACTATAGCAGTTGATGAGAAG TTAAAAAAGCT-3' \\ OHE55: 5 '-GTTATCAGACCATGGGAGTTAAGCTTTTTTAAC TTC-3'}

\section{Minimal $C^{\prime} / D^{\prime}$ K-turn mutant \\ OHE141: $\quad$ 5'-GTAATACGACTCACTATAGAAGCGATGGATGA GCTT-3'}

\section{In vitro transcription of wild-type and mutant sR1}

Uniformly labeled transcripts used in band shift assays were generated by run-off transcription with the T7 RNA polymerase (Invitrogen), in the presence of $13 \mu \mathrm{M}$ GTP and $10 \mu \mathrm{Ci}$ $\left[\alpha-{ }^{32} \mathrm{P}\right]$ GTP (Dupont NEN Research), as previously described in Omer et al. (2002). To generate unlabeled transcripts used in the in vitro methylation assays, the Mega-Short Script kit (Ambion) was used as recommended by the manufacturer.

A clone (pPD1260) containing the $S$. acidocaldarius sR1 cDNA inserted between the EcoRI and BamHI sites of plasmid pGEM $3 \mathrm{Zf}(+)$ was linearized with BamHI and used as template in a T7 RNA polymerase transcription reaction to generate the wild-type sR1 sRNA, from S. acidocaldarius (accession no. AF195095). To generate the target RNA, a 112-bp fragment of rDNA spanning position U52 was amplified from $S$. acidocaldarius genomic DNA using primers AO63.1 and AO65 and then used as template in a transcription reaction using the T7 RNA polymerase. For the sR1 mutants, pairs of oligonucleotides (as indicated above) complementary at their $3^{\prime}$-ends and containing the mutated C/D and/ or C'/D' motifs were annealed and "filled-in" with Klenow DNA polymerase (Invitrogen) following the manufacturer's instructions. The Klenow products were then amplified by PCR, gelpurified, and used as templates in run-off transcription reactions.

\section{Cloning, expression, and purification of recombinant proteins}

The genes encoding the S. solfataricus proteins L7Ae, Nop5, and Fib (accession nos. S75397, NP_342426, P58032) were amplified by PCR, cloned between the NcoI and BamHI sites of pET3d (L7Ae, Fib) or NcoI and EcoRI sites of pET28a (Nop5), and transformed in Escherichia coli. The recombinant proteins were thermoprecipitated and further purified by ion exchange and size exclusion chromatography as described by Ziesche et al. (2004).

\section{Gel retardation assay}

Gel retardation assays were performed as previously described (Omer et al. 2002). Briefly, uniformly labeled wild-type or mutant sR1 transcripts $(0.3 \mathrm{pmol})$ were incubated in binding buffer (25 mM phosphate buffer at $\mathrm{pH} \mathrm{7,100} \mathrm{mM} \mathrm{NaCl}$, and either $1 \mathrm{mM}$ (low) or $10 \mathrm{mM}$ (high) $\mathrm{MgCl}_{2}$ ) in the absence of protein or with a constant amount of 6 pmol of each L7Ae, Nop5, and Fib protein. The reactions were incubated at $70^{\circ} \mathrm{C}$ for $10 \mathrm{~min}$, and $1 \mu \mathrm{L}$ of loading buffer ( $1 \%$ bromophenol blue, $10 \%$ glycerol) was added to the reaction; protein-RNA complexes were resolved on a nondenaturing $6 \%$ polyacrylamide gel containing $0.5 \times \mathrm{TBE}$ buffer and visualized by autoradiography (Omer et al. 2002).

\section{In vitro methylation assay}

For the in vitro methylation assay, equimolar amounts (120 pmol) of guide and target RNA were mixed in $20 \mu \mathrm{L}$ final volume in $25 \mathrm{mM}$ phosphate buffer ( $\mathrm{pH} 7), 100 \mathrm{mM} \mathrm{NaCl}$, denatured by incubating for $1 \mathrm{~min}$ at $95^{\circ} \mathrm{C}$, and renatured by cooling rapidly to $55^{\circ} \mathrm{C}$. The RNAs were added at $0^{\circ} \mathrm{C}$ to an $80-\mu \mathrm{L}$ mixture containing binding buffer supplemented with either $1 \mathrm{mM}$ or $10 \mathrm{mM} \mathrm{MgCl} 2$, [methyl- ${ }^{3} \mathrm{H}$ ] S-adenosyl methionine (300 pmol, $3.9 \mathrm{Ci} / \mathrm{mmol}$; Amersham Pharmacia) and L7Ae, Nop5, and Fib (6 pmol of each). Aliquots $(20 \mu \mathrm{L})$ were removed and transferred to $70^{\circ} \mathrm{C}$ after $2,10,15,20$, and $30 \mathrm{~min}$. The individual $20-\mu \mathrm{L}$ samples were placed on ice and precipitated with $5 \%$ trichloroacetic acid; the precipitates were collected on $0.2 \mu \mathrm{m}$ nitrocellulose filters (Millipore) and dried, and radioactivity was measured by scintillation counting (Omer et al. 2002).

\section{ACKNOWLEDGMENTS}

This work was supported by the Natural Sciences and Engineering Research Council, the Canadian Institute for Health Research, and the National Science Foundation. The opinions, findings, and conclusions expressed in this publication are those of the authors and do not necessarily reflect the views of the National Science Foundation.

Received January 21, 2006; accepted May 30, 2006.

\section{REFERENCES}

Aittaleb, M., Rashid, R., Chen, Q., Palmer, J.R., Daniels, C.J., and Li, H. 2003. Structure and function of archaeal box C/D sRNP core proteins. Nat. Struct. Biol. 10: 256-263.

Bachellerie, J.P. and Cavaille, J. 1997. Guiding ribose methylation of rRNA. Trends Biochem. Sci. 22: 257-261.

Bachellerie, J.P., Nicoloso, M., Qu, L.H., Michot, B., CaierguesFerrer, M., Cavaille, J., and Renalier, M.H. 1995. Novel intronencoded small nucleolar RNAs with long sequence complementarities to mature rRNAs involved in ribosome biogenesis. Biochem. Cell Biol. 73: 835-843.

Bortolin, M.L., Bachellerie, J.P., and Clouet-d'Orval, B. 2003. In vitro RNP assembly and methylation guide activity of an unusual box C/D RNA, cis-acting archaeal pre-tRNA ${ }^{\text {Trp }}$. Nucleic Acids Res. 31: 6524-6535.

Cahill, N.M., Friend, K., Speckmann, W., Li, Z.H., Terns, R.M., Terns, M.P., and Steitz, J.A. 2002. Site-specific cross-linking analyses reveal an asymmetric protein distribution for a box C/D snoRNP. EMBO J. 21: 3816-3828. 
Dennis, P.P. and Omer, A. 2005. Small non-coding RNAs in Archaea. Curr. Opin. Microbiol. 8: 685-694.

Filipowicz, W., Pelczar, P., Pogacic, V., and Dragon, F. 1999. Structure and biogenesis of small nucleolar RNAs acting as guides for ribosomal RNA modification. Acta Biochim. Pol. 46: 377-389.

Gerbi, S.A. 1995. Small nucleolar RNA. Biochem. Cell Biol. 73: 845858.

Gott, J.M., Pan, T., LeCuyer, K.A., and Uhlenbeck, O.C. 1993. Using circular permutation analysis to redefine the R17 coat protein binding site. Biochemistry 32: 13399-13404.

Hamma, T. and Ferre-D'Amare, A.R. 2004. Structure of protein L7Ae bound to a K-turn derived from an archaeal box H/ACA sRNA at $1.8 \AA$ A resolution. Structure 12: $893-903$.

Harris, M.E. and Pace, N.R. 1995. Analysis of the tertiary structure of bacterial RNase P RNA. Mol. Biol. Rep. 22: 115-123.

Hernandez-Verdun, D. 2005. Nucleolus: From structure to dynamics. Histochem. Cell Biol. 125: 127-137.

Kiss, T. 2001. Small nucleolar RNA-guided post-transcriptional modification of cellular RNAs. EMBO J. 20: 3617-3622.

Klein, D.J., Schmeing, T.M., Moore, P.B., and Steitz, T.A. 2001. The kink-turn: A new RNA secondary structure motif. EMBO J. 20: 4214-4221.

Kuhn, J.F., Tran, E.J., and Maxwell, E.S. 2002. Archaeal ribosomal protein L7 is a functional homolog of the eukaryotic $15.5 \mathrm{kD} /$ Snu13p snoRNP core protein. Nucleic Acids Res. 30: 931-941.

Lafontaine, D. and Tollervey, D. 1995. Trans-acting factors in yeast pre-rRNA and pre-snoRNA processing. Biochem. Cell Biol. 73: 803-812.

Matsumura, S., Ikawa, Y., and Inoue, T. 2003. Biochemical characterization of the kink-turn RNA motif. Nucleic Acids Res. 31: $5544-5551$.

Maxwell, E.S. and Fournier, M.J. 1995. The small nucleolar RNAs. Annu. Rev. Biochem. 64: 897-934.

Moore, T., Zhang, Y., Fenley, M.O., and Li, H. 2004. Molecular basis of box C/D RNA protein interactions; cocrystal structure of archaeal L7Ae and a box C/D RNA. Structure 12: 807-818.

Nolivos, S., Carpousis, A.J., and Clouet-d'Orval, B. 2005. The K-loop, a general feature of the Pyrococcus C/D guide RNAs, is an RNA structural motif related to the K-turn. Nucleic Acids Res. 33: 65076514.

Nottrott, S., Urlaub, H., and Luhrmann, R. 2002. Hierarchical, clustered protein interactions with U4/U6 snRNA: A biochemical role for U4/U6 proteins. EMBO J. 21: 5527-5538.

Omer, A.D., Lowe, T.M., Russell, A.G., Ebhardt, H., Eddy, S.R., and Dennis, P.P. 2000. Homologs of small nucleolar RNAs in Archaea. Science 288: $517-522$.
Omer, A.D., Ziesche, S., Ebhardt, H., and Dennis, P.P. 2002. In vitro reconstitution and activity of a C/D box methylation guide ribonucleoprotein complex. Proc. Natl. Acad. Sci. 99: 5289-5294.

Pan, T., Gutell, R.R., and Uhlenbeck, O.C. 1991. Folding of circularly permuted transfer RNAs. Science 254: 1361-1364.

Pan, T., Fang, X., and Sosnick, T. 1999. Pathway modulation, circular permutation and rapid RNA folding under kinetic control. J. Mol. Biol. 286: 721-731.

Rashid, R., Aittaleb, M., Chen, Q., Spiegel, K., Demeler, B., and Li, H. 2003. Functional requirement for symmetric assembly of archaeal box C/D small ribonucleoprotein particles. J. Mol. Biol. 333: 295-306.

Suryadi, J., Tran, E.J., Maxwell, E.S., and Brown II, B.A. 2005. The crystal structure of the Methanocaldococcus jannaschii multifunctional L7Ae RNA-binding protein reveals an induced-fit interaction with the box C/D RNAs. Biochemistry 44: 9657-9672.

Szewczak, L.B., Gabrielsen, J.S., Degregorio, S.J., Strobel, S.A., and Steitz, J.A. 2005. Molecular basis for RNA kink-turn recognition by the h15.5K small RNP protein. RNA 11: 1407-1419.

Tollervey, D. and Kiss, T. 1997. Function and synthesis of small nucleolar RNAs. Curr. Opin. Cell Biol. 9: 337-342.

Tran, E.J., Zhang, X., and Maxwell, E.S. 2003. Efficient RNA 2'-Omethylation requires juxtaposed and symmetrically assembled archaeal box C/D and $C^{\prime} / \mathrm{D}^{\prime}$ RNPs. EMBO J. 22: 3930-3940.

Tran, E., Zhang, X., Lackey, L., and Maxwell, E.S. 2005. Conserved spacing between the box C/D and $\mathrm{C}^{\prime} / \mathrm{D}^{\prime}$ RNPs of the archaeal box C/D sRNP complex is required for efficient 2'-O-methylation of target RNAs. RNA 11: 285-293.

Vidovic, I., Nottrott, S., Hartmuth, K., Luhrmann, R., and Ficner, R. 2000. Crystal structure of the spliceosomal $15.5 \mathrm{kD}$ protein bound to a U4 snRNA fragment. Mol. Cell 6: 1331-1342.

Watkins, N.J., Segault, V., Charpentier, B., Nottrott, S., Fabrizio, P., Bachi, A., Wilm, M., Rosbash, M., Branlant, C., and Luhrmann, R. 2000. A common core RNP structure shared between the small nucleolar box C/D RNPs and the spliceosomal U4 snRNP. Cell 103: $457-466$.

Watkins, N.J., Dickmanns, A., and Luhrmann, R. 2002. Conserved stem II of the box C/D motif is essential for nucleolar localization and is required, along with the $15.5 \mathrm{~K}$ protein, for the hierarchical assembly of the box C/D snoRNP. Mol. Cell. Biol. 22: 8342-8352.

Weinstein, L.B. and Steitz, J.A. 1999. Guided tours: From precursor snoRNA to functional snoRNP. Curr. Opin. Cell Biol. 11: 378-384.

Woodson, S.A. 2002. Folding mechanisms of group I ribozymes: Role of stability and contact order. Biochem. Soc. Trans. 30: 1166-1169.

Ziesche, S.M., Omer, A.D., and Dennis, P.P. 2004. RNA-guided nucleotide modification of ribosomal and non-ribosomal RNAs in Archaea. Mol. Microbiol. 54: 980-993. 

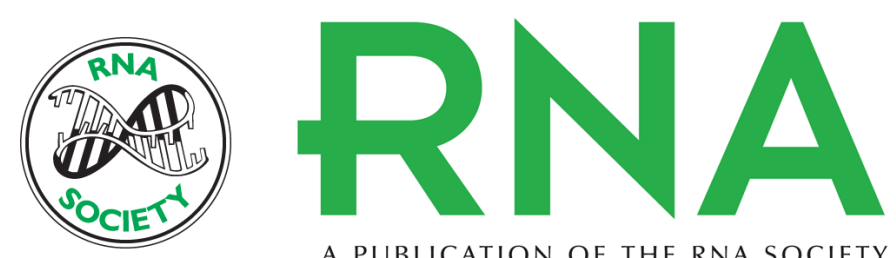

A PUBLICATION OF THE RNA SOCIETY

\section{Probing the structure and function of an archaeal C/D-box methylation guide sRNA}

Arina D. Omer, Maria Zago, Alex Chang, et al.

RNA 2006 12: 1708-1720

References This article cites 37 articles, 11 of which can be accessed free at:

http://rnajournal.cshlp.org/content/12/9/1708.full.html\#ref-list-1

License

Email Alerting Receive free email alerts when new articles cite this article - sign up in the box at the Service top right corner of the article or click here.

To subscribe to RNA go to:

http://rnajournal.cshlp.org/subscriptions 